\title{
Correlation between inner strength and health-promoting behaviors in women with heart failure
}

\author{
Meimanat Hosseini ${ }^{1}$, Parvaneh Vasli ${ }^{1}$, Sakineh Rashidi ${ }^{2}$, Soodeh Shahsavari ${ }^{3}$
}

\author{
${ }^{1}$ Ph.D., Assistant Professor, Department of Community Health Nursing, School of Nursing and Midwifery, Shahid \\ Beheshti University of Medical Sciences, Tehran, Iran \\ ${ }^{2}$ MSN, School Of Nursing and Midwifery, Shahid Beheshti University of Medical Sciences, Tehran, Iran \\ ${ }^{3}$ Ph.D., Assistant Professor, Health Information Management Department, Faculty of Paramedical Sciences, \\ Kermanshah University of Medical Sciences, Kermanshah, Iran
}

\section{Type of article: Original}

\begin{abstract}
Introduction: Inner strength is a factor for mental health and well-being and, consequently, a dynamic component of holistic healing. Health-promoting behaviors are appropriate activities to improve health status and prevent the progression of the functional defect resulting from heart failure. The present study aimed to determine the correlation between inner strength and health-promoting behaviors in women with heart failure referred to hospitals affiliated with Shahid Beheshti University of Medical Sciences (SBMU) in 2013.

Methods: In this cross-sectional study, 145 women with hearth failure were selected through convenient sampling from the clients referred to hospitals affiliated with SBMU. The data collection tool included a threesection questionnaire of personal characteristics, inner strength, and health-promoting life profile II (HPLP II). The data analysis used descriptive statistical tests and Pearson correlation coefficient through SPSS version 20.

Results: A direct significant correlation was found between inner strength and all dimensions of healthpromoting behaviors and overall health-promoting behaviors $(\mathrm{p}=0.000)$ as well as between all dimensions of inner strength (except for the dimension of knowing and searching with physical activity and the dimension of connectedness with personal accountability in healthcare as well as connectedness with physical activity) with health-promoting behaviors ( $\mathrm{p}=0.000$ to $\mathrm{p}=0008)$.

Conclusion: To improve the level of health and well-being and reduce the costs of care services in women with health failure, close attention should be paid to developing and empowering their inner strength.

Keywords: Inner strength, Health-promoting behaviors, Heart failure, Women
\end{abstract}

\section{Introduction}

Chronic heart failure is a progressive disabling disorder (1). The number of the patients with heart failure is increasing in both developed and developing countries, with approximately $1 \%-2 \%$ of adults in developed countries experiencing heart failure (2). In Iran, the number of these patients is estimated to be about 3,500 out of 100,000 (3). Meanwhile, about $50 \%-55 \%$ of the patients with heart failure are women (4), although most studies consider women less often (5). Women's experience of heart failure is different than that of men in some aspects. Framingham's heart study showed that women report a higher effect of heart failure on their daily lives, and they also have a lower quality of life compared to men (6). In fact, heart failure, with its potentially disabling nature, can affect daily life activities, and these patients lose their independency in their daily life activities and must rely on others for their self-care (7). In this way, individuals' ability to be involved in health-promoting behaviors as appropriate activities to improve health status and prevent the severe functional defect of the disease is influenced (6). Health-promoting behaviors are activities to maintain or increase the level of health and self-actualization and happiness among individuals (7). These behaviors emphasize a positive pattern of life, which leads to an increase in health level and

\section{Corresponding author:}

Assistant Professor Dr. Parvaneh Vasli, Department of Community Health Nursing, School of Nursing and Midwifery, Shahid Beheshti University of Medical Sciences, Tehran, Iran.

Tel: +98.2188655368, Email: parvanehvasli@yahoo.com and p-vasli@sbmu.ac.ir

Received: February 06, 2016, Accepted: May 19, 2016, Published: August 2016

iThenticate screening: May 19, 2016, English editing: June 26, 2016, Quality control: July 06, 2016

(C) 2016 The Authors. This is an open access article under the terms of the Creative Commons Attribution-NonCommercialNoDerivs License, which permits use and distribution in any medium, provided the original work is properly cited, the use is non-commercial and no modifications or adaptations are made. 
quality of life (8). Pender believes that healthy life behaviors include spiritual growth; personal responsibility for health, sports, and nutrition; interpersonal communications; and tension management (9). The effectiveness of health-promoting behaviors on the promotion of individuals' health has been confirmed in various studies (10). In fact, these behaviors should be considered the main strategies for maintaining and improving health (11). Enjezab et al.'s study of health-promoting behaviors of middle-aged women in Yazd showed a low level of these behaviors in components of physical activity and personal responsibility for health (12). Thanavaro, Thanavaro, and Delicath, in their study of women with chest pain (13), and Kheirjoo et al., in their study of women with rheumatoid arthritis, also reported that health-promoting behaviors were not appropriate among these women (14). On the other hand, physical and mental health are considered mutually dependent and integrated. Inner strength is a factor for mental health and emotional well-being (15) and, consequently, a dynamic component of holistic healing (16). People have the resources to survive, grow, and mature just as they have the abilities to resist adversities, endure suffering, and experience a good life despite harsh conditions. The ways people succeed in facing difficulties, such as prolonged illness, functional decline, grief, and loss, vary according to life circumstances and personal capacities and other resources. Inner strength has been defined as an inner source of promoting well-being in human beings (17). Inner strength contains four concepts: "knowing or anguish and searching," "connectedness," "engagement," and "movement" (18). Knowing and searching refers to passing over fear, vulnerability, and weakness to move from a feeling of fear and shock toward acceptance. Connectedness is described as the development of supporting selfcommunication as well as communication with family and friends, and it acts as a spiritual power. Engagement refers to self-construction and enjoyment of life facilities. Finally, movement describes the dimension of movement, rest, activity, an honest self-evaluation, and physical and mental balance (19). All humans have the potential and capacity to construct and empower themselves through inner strength (16). Inner strength exists in humans prior to the occurrence of challenging life events, such as chronic diseases, and a life-changing event or challenge can trigger its manifestation (18). Putnam studied the association between inner strength and health-promoting behaviors and its effects on quality of life among middle-aged women, reporting a positive association between these two factors. Inner strength and health-promoting behaviors were also determined as predictive elements for quality of life (20). In another study by Dingley on inner strength in women with cancer, a strong association between inner strength and self-management was observed among patients (19). Nursing, as a profession, is committed to promoting health among society's members. The holistic approach in nursing is a reason for considering a human as a unique existence; as such, one's physical, psychological, and mental health are not separable as they are combined in an integrated entity. Well-being and various aspects of human health have a multi-dimensional and interactive association with each other (21). The effective role of inner strength and health-promoting behaviors on the improvement of quality of life and obtaining of positive health results and self-management in chronic diseases has been demonstrated (20). Heart failure is one of the most fatal diseases in Iranian women (22), who play important roles by fulfilling their daily activities; indeed, female seniors are more active than their male counterparts (23). For these reasons, in the last two decades, increasing efforts have been made to evaluate and promote the well-being of Iranian women with heart failure; however, little is known in this regard (22). Thus, it seemed necessary to conduct a study related to subjects such as inner strength and health-promoting behaviors in women with heart failure in Iranian society. This study aimed to determine the correlation between inner strength and health-promoting behaviors in women with heart failure referred to the hospitals affiliated with Shahid Beheshti University of Medical Sciences (SBMU) in Tehran, the capital of Iran. Because these hospitals are affiliated with one of the most important and largest universities for education, treatment, and research in medical sciences, patients throughout the country are referred to them, including patients with heart diseases.

\section{Material and Methods}

\subsection{Research design and setting}

In this cross-sectional study, five hospitals affiliated with SBMU with active cardiac clinics were selected through purposive sampling. The data gathering was conducted in June 2013. One hundred forty-five qualified women with heart failure were included in the study through convenient sampling.

\subsection{Inclusion and exclusion criteria}

Inclusion criteria were age $\geq 40$ years, a diagnosed recorded heart failure in the patient' $s$ file and medical records, class two or three heart failure, at least six weeks since disease diagnosis, ability to read and write in Persian, and no history of mental diseases or the use of psychotropic medications. Patients who changed their minds about completing the questionnaires while completing them were excluded from the study. 


\subsection{Instrument}

The data collection tool included three questionnaires. The first was a personal characteristics questionnaire, a researcher-made 12-item questionnaire in which 11 items asked about age, marital status, level of education, spouse's level of education, number of children, occupation status, family monthly income, medical insurance coverage status, length of heart failure involvement, number of hospitalizations in the past year, and involvement in other physical diseases. Participants completed these 11 items; the remaining item, on the classification of the disease, was completed by the researcher. The second tool was a 27 -item tool measuring inner strength in women with chronic diseases, designed by Roux et al. in 2003 (20). This tool investigates the dimensions of inner strength in four dimensions of knowing and searching with seven items, connectedness with seven items, engagement with six items, and movement with seven items (16) based on a five-point scale (i.e., absolutely agree, agree, somewhat agree, disagree, and absolutely disagree). Possible points ranged from 27 to 135, with higher points showing higher inner strength (19). The health-promoting life profile II (HPLP II), the third tool, is a 52-item questionnaire designed by Walker et al. in 1987 based on Pender's health-promoting model (11). This tool measures the application of health-promoting behaviors in six subscales: nutrition (nine items), physical activity (eight items), personal accountability in healthcare (nine items), tension management (eight items), interpersonal communications (nine items), and spiritual growth (nine items). Possible responses were never, sometimes, usually, and always. The points of health-promoting behavior range from 52 to 208 and can be separately calculated for each dimension (24).

\subsection{Validity and reliability}

The content validity index and content validity ratio and face validity were used to measure the tools' validity in such way that the three-section questionnaires were distributed among three psychiatric nurses, three public health nurses, and four PhDs of nursing, all of whom were academic members of nursing schools of Tehran Universities of Medical Sciences (TUMS) and SBMU. Validity was confirmed by 10 experts. Content validity indexes for the inner strength questionnaire concerning association (0.97), simplicity (0.96), and clarity (0.97) were obtained. The content validity index for the whole HPLP II concerning association, simplicity, and clarity was calculated as 0.98 . The content validity ratio for inner strength and HPLP II questionnaires were calculated as 0.93 and 0.95 , respectively. Experts' viewpoints of the content validity calculation were used to check the face validity of the questionnaire. In addition, viewpoints of five qualified patients concerning the understandability and appearance of appropriateness of the items were also considered. Two methods of internal consistency, Cronbach's alpha and test-re-test, were used to calculate reliability. Cronbach's alphas of inner strength questionnaire and HPLP II were $\alpha=0.89$ and $\alpha=0.83$, respectively. To conduct test-re-test, 15 qualified patients were selected and three questionnaires were given to them with a two-week interval and after completion. The Pearson correlation coefficient for the inner strength questionnaire and HPLP II were calculated as $\mathrm{r}=0.87$ and $\mathrm{r}=0.89$, respectively.

\subsection{Data collection}

To collect data, after getting written permission from the post-graduate department of SBMU, one of the researchers referred to the selected hospitals and obtained written permission from the hospital authorities. She then referred to the hospital clinics and, after introducing herself and explaining the research goals to the head of the clinic as well as coordinating with her, referred to female patients' medical files in the cardiology clinic, identifying those patients with a recorded heart failure diagnosis in their files. Finally, the qualified subjects were selected through convenient sampling. Due to a lack of precise statistics for female patients with heart failure and given that there was no possibility of other sampling methods, convenient sampling was used. Written consent was obtained from the subjects to participate in the study. For subjects' convenience and to achieve more precise information from them, a quiet place in the clinic was considered for the completion of the questionnaires. For those subjects with lower educational level, further explanations were given, as needed. Data were analyzed using descriptive statistical tests and the Pearson correlation coefficient to determine the association between data via IBM SPSS Statistics version 20 (IBM Corp., Armonk, NY, USA). The 0.01 significance level was considered to obtain more accurate estimates.

\section{Results}

The results showed that the mean age of subjects was 57.46 years. Approximately $89.6 \%$ of the subjects were married, and $54.5 \%$ and $35.9 \%$ of subjects and their spouses, respectively, had primary school education. Subjects' mean number of children was 3.47 (1.61), $78.6 \%$ of the subjects were homemakers, and $45.5 \%$ earned a family monthly income between 33 and 165 U.S. dollars. Approximately $93.8 \%$ had medical insurance, and $51.7 \%$ and $48.3 \%$ were in the second and third classes of the disease, respectively. Subjects' mean length of heart failure was 15.29 (10.72) months, $51.7 \%$ had a history of hospitalization in the past year, and $48.3 \%$ had other physical diseases in addition to heart failure. The data also showed that $65.5 \%$ of the subjects had appropriate inner strength, $32.4 \%$ 
had moderate, and $2.1 \%$ had poor inner strength. Means (SD) of obtained points in inner strength and its subscales are presented in Table 1. In addition, the results showed that $54.5 \%$ of the subjects had a moderate level of healthpromoting behaviors and $4.1 \%$ had a poor level of health-promoting behaviors. None of the subjects had excellent health-promoting behaviors. Means (SD) of calculated points in health-promoting behaviors and its subscales are presented in Table 2. The Pearson correlation coefficient showed a direct and significant correlation between the variable of inner strength and general promotion behaviors and all dimensions of health-promoting behaviors (Table 3 ). The correlation of inner strength with health-promoting behaviors was $r=0.77$, revealing a direct correlation between two variables in such way that an increase or decrease in the inner strength variable would change healthpromoting behaviors in the same way $(\mathrm{p}=0.000)$. The data also showed that all dimensions of inner strength (except for knowing and searching with physical activity, the dimension of connectedness with personal accountability in healthcare, and the dimension of connectedness with physical activity) had a direct significant association with health-promoting behaviors $(\mathrm{p}=0.000$ to $\mathrm{p}=0.008$ ) (Table 4).

Table 1. Range of points, mean, and SD of inner strength and its subscales in women with heart failure

\begin{tabular}{|l|l|l|l|l|}
\hline Variable & Points range & Obtained point range & Mean & SD \\
\hline Inner strength & $27-135$ & $48-125$ & 92.60 & 19.26 \\
\hline Knowing and searching & $7-35$ & $16-34$ & 21.26 & 4.43 \\
\hline Connectedness & $7-35$ & $11-34$ & 24.85 & 5.44 \\
\hline Engagement & $6-30$ & $8-28$ & 21.26 & 5.19 \\
\hline Movement & $7-35$ & $9-32$ & 21.11 & 6.40 \\
\hline
\end{tabular}

Table 2. Range of points, mean, and SD of health-promoting behaviors and its subscales in women with heart failure

\begin{tabular}{|l|l|l|l|l|}
\hline Variable & Point range & Obtained point range & Mean & SD \\
\hline Health-promoting behaviors & $1-4$ & $1.66-3.03$ & 2.40 & 0.35 \\
\hline Health accountability & $1-4$ & $1.44-3.56$ & 2.50 & 0.46 \\
\hline Physical activity & $1-4$ & $1-2.63$ & 1.19 & 0.27 \\
\hline Nutrition & $1-4$ & $1.67-3.56$ & 2.58 & 0.39 \\
\hline Spiritual growth & $1-4$ & $1.22-4$ & 2.84 & 0.77 \\
\hline Interpersonal communications & $1-4$ & $1.44-3.78$ & 2.87 & 0.57 \\
\hline Tension management & $1-4$ & $1.50-3.50$ & 2.44 & 0.45 \\
\hline
\end{tabular}

Table 3. Results of correlation between inner strength and health-promoting behaviors in women with heart failure

\begin{tabular}{|l|l|l|}
\hline Variable & Pearson correlation coefficient & p-value \\
\hline Health accountability & 0.34 & 0.000 \\
\hline Physical activity & 0.30 & 0.000 \\
\hline Nutrition & 0.33 & 0.000 \\
\hline Spiritual growth & 0.83 & 0.000 \\
\hline Interpersonal communications & 0.53 & 0.000 \\
\hline Tension management & 0.73 & 0.000 \\
\hline Total health-promoting behaviors & 0.77 & 0.000 \\
\hline
\end{tabular}

Table 4. Results of correlation between dimensions of inner strength and health-promoting behaviors in women with heart failure

\begin{tabular}{|l|l|l|l|l|}
\hline \multirow{2}{*}{ Health-promoting behaviors dimensions } & \multicolumn{4}{l|}{ Inner strength dimensions $(\mathrm{r}, \mathrm{p})$} \\
\cline { 2 - 5 } & Knowing and searching & Connectedness & Engagement & Movement \\
\hline Health accountability & $0.32,0.000$ & $0.19,0.022$ & $0.36,0.000$ & $0.34,0.000$ \\
\hline Physical activity & $0.18,0.033$ & $0.19,0.020$ & $0.36,0.001$ & $0.33,0.001$ \\
\hline Nutrition & $0.22,0.008$ & $0.24,0.003$ & $0.36,0.001$ & $0.34,0.001$ \\
\hline Spiritual growth & $0.61,0.001$ & $0.73,0.001$ & $0.78,0.001$ & $0.82,0.001$ \\
\hline Interpersonal communications & $0.61,0.001$ & $0.40,0.001$ & $0.53,0.001$ & $0.54,0.001$ \\
\hline Tension management & $0.54,0.001$ & $0.61,0.001$ & $0.69,0.011$ & $0.73,0.001$ \\
\hline
\end{tabular}




\section{Discussion}

This study examined the correlation between inner strength and health-promoting behaviors in women with heart failure referred to hospitals affiliated with SBMU. The results showed that the mean score of inner strength in women with heart failure was 92.6 (18.25), indicating an appropriate level. Dingley (19) reported a mean score of inner strength as 108.6 in women with breast cancer, which shows an excellent level. Putnam reported a mean score of inner strength as 56.86 in middle-aged women, indicating a moderate level (20). Roux, Dingley, and Bush concluded that an important event in life can lead to the manifestation of inner strength (21). Inner strength exists prior to the occurrence of a challenge in life, and experiencing challenging events triggers the potential for and ability to access inner strength. In the present study and in Dingley's (19) study, subjects experienced a specific challenge (onset of the disease) that acted as an effective factor in the development of inner strength and caused their inner strength to reach an appropriate level. Meanwhile, in Putnam's study, about three-fourths of participants were completely healthy and, consequently, could facilitate inner strength development (20). Among inner strength dimensions, subjects obtained higher points on the dimension of connectedness, which is consistent with Dingley's results (19). Connectedness shows a deep relationship with God, family, and friends. Obtaining higher points on the dimension of connectedness among subjects can be due to the fact that, in cases of a disease, missing someone, sorrow, or a great change in life, individuals seek help from spiritual resources to adapt or fulfill their needs (25). In Putnam's study, participants obtained the lowest points on the dimension of connectedness (20). This difference in results can be due to the cultural and religious differences in various societies. In Iranian society, religion, spirituality, and one's spiritual relationship with God and the family as well as familial communications play a major role in individuals' lives. Iranians always-and especially in cases of problems-get support from their religious beliefs as an important source. On the other hand, family members try to make closer relationships with the member in trouble in order to solve the problem. The lowest obtained points on dimensions of inner strength were for movement, which might be due to women's limited knowledge of heart failure and the advantages of appropriate physical activity and movement. Subjects' high fear of worsening their condition through physical activity as well as their limited familiarity with relaxation activities, resulting in a physical and mental balance in the body, could be other reasons.

In this study, $65.5 \%$ of subjects indicated an appropriate level of inner strength while other subjects had moderate and poor levels. In fact, not all of the individuals were necessarily empowered by inner strength after being exposed to life-challenging events like a chronic disease. This inner source of human strength is influenced by personal, external, and environmental factors (16). Empowering patients' inner strength is achieved by helping them maintain a relationship with their family and friends and preserve a spiritual relationship as well as inquiring about the disease and having access to resources to answer the questions. Encouraging patients to express their fears and detect their inner power, collaborating in decision making concerning treatment programs, accessing supportive resources, and focusing on achieving health instead of on the disease are other factors that help empower inner strength. In the present study, $34.5 \%$ of the subjects had a moderate or poor level of inner strength, revealing the need for nurses', authorities', and healthcare providers' attention to help women with heart failure detect, maintain, and empower their inner strength. Meanwhile, in Enjezab et al.'s study, middle-aged women in Yazd obtained higher points in health-promoting behaviors (12). Their participants were selected through cluster sampling from middle-aged women in Yazd. Although the inclusion criteria were a lack of any mental or speech problems, the participants' health status was not determined whereas subjects in the present study had heart failure. As individuals' health status affects the frequency of their participation in health-promoting behaviors, the difference between Enjezab et al.'s study and the present study seem reasonable (12). Pierce's study on women with heart failure (6) and Beal et al.'s study on women with fibromyalgia (26) revealed an appropriate level of health-promoting behaviors in these women. This difference could stem from socio-cultural difference between developing and developed countries. The difference in the level of health-promoting behaviors in various societies can result from personal, economic, social, and environmental factors, which affect individuals' health status. Social norms, mass media, national health policies, commercial activities, and environmental conditions affect individuals' health-promoting behaviors (11). Unfortunately, in Iran, health-promoting programs have not found their appropriate place in health and treatment services, yet most of the budget in this field is spent on treatment and medication (27).

Based on the results of the present study, subjects obtained the highest point in interpersonal communications. In most studies conducted on lifestyles, high points have been reported for interpersonal support in both healthy individuals and those with chronic diseases (14). Based on their obtained points, the subjects were also at an appropriate level for the dimensions of spiritual growth and nutrition and at a moderate level for the dimensions of personal accountability in healthcare and tension management, which is in line with Pierce's study on women with 
heart failure (6). Kheirjoo et al. (14) examined on women with rheumatoid arthritis (14) and Bea et al. examined women with fibromyalgia (26)' in both studies, dimensions of spiritual growth, nutrition, and personal accountability in healthcare were at an appropriate level while the dimension of tension management was at a moderate level. With regard to the moderate level of personal accountability in healthcare among subjects in the present study, personal accountability in healthcare refers to the acceptance of the responsibility for one's health and administration of self-care. Some patients with heart failure actually do not believe in the positive effect of their selfcare behaviors in relieving their disease symptoms, which impairs their self-care behaviors; these patients have no motivation for such behaviors (28). In the present study, inner strength had a direct significant correlation with general health-promoting behaviors as well as all its dimensions, which is in line with Putnam's (20) finding. Inner strength is a factor for mental health and psychological well-being and a component of spirituality and is also considered as a dynamic element in holistic healing. The ability to face challenges and facilitate healing, make an appropriate change in life, feel self-control over one's condition, and have the possibility to maintain a balance between one's inner world and one's life experiences and the environment are the outcomes of inner strength (16). Stronger inner strength is associated with higher mental and spiritual health. Given these findings, women with heart failure who have stronger inner strength, have a greater potential to adapt to a chronic disease condition and try to reach the highest level of health. Health-promoting behaviors are people's behaviors or activities resulting from their desire to amend their health status (29). The possibility of engaging in such behaviors in women with heart failure who have stronger inner strength is high, and the obtained positive significant association between inner strength and health-promoting behaviors in the subjects is reasonable. The results also showed that all dimensions of inner strength (except for knowing and searching with physical activity and the dimensions of connectedness with personal accountability in healthcare and connectedness with physical activity) have a direct significant correlation on dimensions of health-promoting behaviors. Putnam also found a direct significant correlation between all dimensions of inner strength (expect for knowing and searching with dimensions of personal accountability in healthcare, the dimension of connectedness with the dimension of physical activity, and the dimension of connectedness with the dimension of nutrition) with dimensions of the health-promoting behaviors variable (20), which is in line with the findings of the present study.

\section{Study limitations}

One limitation of this study related to patients' self-reports. Patients' carelessness and mental condition could influence their responses to the questionnaires. Another limitation was the small number of studies on inner strength and health-promoting behaviors involving patients with heart failure in Iran and the world; the authors tried to minimize this limitation by using related studies.

\section{Conclusions}

This study aimed to determine the correlation between inner strength and health-promoting behaviors in women with heart failure. The results showed a direct significant correlation between them. Therefore, to enhance the level of health and well-being and lower the costs of care in women with heart failure, it seems essential for nurses to develop and empower patients' inner strength. Due to the limited number of studies on health promotion for patients with chronic disease, especially female patients in Iran, the authors suggest conducting more studies to complete this line of research.

\section{Acknowledgments:}

This study was extracted from a postgraduate thesis. The study was approved by the Ethics Committee of the SBMU under the code SBMU.REC.1392.357. The authors greatly appreciate the heads of selected hospitals and the patients cooperating with the research.

\section{Conflict of Interest:}

There is no conflict of interest to be declared.

\section{Authors' contributions:}

All authors contributed to this project and article equally. All authors read and approved the final manuscript.

\section{References:}

1) Shafazand M, Patel H, Ekman I, Swedberg K, Schaufelberger M. Patients with worsening chronic heart failure who present to a hospital emergency department require hospital care. BMC Res Notes. 2012; 5: 132. doi: 10.1186/1756-0500-5-132. 
2) McMurray J, Adamopoulos S, Anker SD, Auricchio A, Bohm M, Dickstein K, et al. ESC guidelines for the diagnosis and treatment of acute and chronic heart failure 2012: the task force for the diagnosis and treatment of acute and chronic heart failure 2012 of the European society of cardiology. Developed in collaboration with the heart failure association (HFA) of the ESC. Eur J Heart Fail. 2012; 14(8): 803-69. doi: 10.1093/eurjhf/hfs105. PMID: 22828712.

3) Bahrami M, Etemadifar S, Shahriari M, Farsani AK. Caregiver burden among Iranian heart failure family caregivers: A descriptive, exploratory, qualitative study. Iran J Nurs Midwifery Res. 2014; 19(1): 56-63. PMID: 24554961, PMCID: PMC3917186.

4) Shah RU, Klein L, Lloyd-Jones DM. Heart failure in women: epidemiology, biology and treatment. Women's Health. 2009; 5(5): 517-27. doi: 10.2217/whe.09.50.

5) Ginghina C, Botezatu CD, Serban M, Jurcut R. A personalized medicine target: heart failure in women. J Med Life. 2011; 4(3): 280 - 6. PMID: 22567052, PMCID: PMC3168816.

6) Pierce CS. Health promoting behaviors of rural women with heart failure. Online Journal of Rural Nursing and Health Care. 2005; 5(2): 28-37.

7) Siabani S, Leeder SR, Davidson PM. Barriers and facilitators to self-care in chronic heart failure: a metasynthesis of qualitative studies. Springerplus. 2013; 2: 320. doi: 10.1186/2193-1801-2-320. PMID: 23961394, PMCID: PMC3727080.

8) Peker K, Bermek G. Predictors of health-promoting behaviors among freshman dental students at Istanbul University. J Dent Educ. 2011; 75(3): 413-20. PMID: 21368265

9) Hosseinnejad M, Klantarzadeh M. Study of lifestyle based on the Pender's Health Promotion model among students of Islamic Azad University. Journal of Health Education and Health Promotion. 2014; 1(4): 15-28.

10) Harooni J, Hassanzadeh A, Mostafavi $F$. Influencing factors on health promoting behavior among the elderly living in the community. J Educ Health Promot. 2014; 3: 40. doi: 10.4103/2277-9531.131921. PMID: 25013833, PMCID: PMC4089138.

11) Baheiraei A, Mirghafourvand M, Mohammadi E, Nedjat S, Charandabi SM, Rajabi F, et al. Healthpromoting behaviors and social support of women of reproductive age, and strategies for advancing their health: Protocol for a mixed methods study. BMC Public Health. 2011; 11(1): 191-5. doi: 10.1186/14712458-11-191.

12) Enjezab B, Farajzadegan Z, Taleghani F, Aflatoonian A, Morowatisharifabad MA. Health Promoting Behaviors in a Population-based Sample of Middle-aged Women and its Relevant Factors in Yazd, Iran. Int J Prev Med. 2012; 3(1): 191-8. PMID: 22826765, PMCID: PMC3399308.

13) Thanavaro JL, Thanavaro S, Delicath T. Health promotion behaviors in women with chest pain. Heart Lung. 2010; 39(5): 394-403. doi: 10.1016/j.hrtlng.2009.10.016. PMID: 20561847.

14) Kheirjoo E, Jomehri F, Ahadi H, Farshbaf Manisefat F. Comparison of health promoting lifestyle of female Rheumatoid Arthritis patients with healthy women and its relationship with demographic factors. Knowledge and Research in Applied Psychology. 2013; 13(4): 61-70.

15) Dingley C, Roux G. The role of inner strength in quality of life and self-management in women survivors of cancer. Research in Nursing \& Health. 2014; 37(1): 32-41. doi: 10.1002/nur.21579.

16) Lewis KL, Roux G. Psychometric testing of the Inner Strength Questionnaire: women living with chronic health conditions. Appl Nurs Res. 2011; 24(3): 153-60. doi: 10.1016/j.apnr.2009.06.003. PMID: 21777790.

17) Alpers LM, Helseth S, Bergbom I. Experiences of inner strength in critically ill patients: A hermeneutical approach. Intensive Crit Care Nurs. 2011; 28(3): 150-8. doi: 10.1016/j.iccn.2011.10.004. PMID: 22225817.

18) Orshan SA, Ventura JL, Covington SN, Vanderhoof VH, Troendle JF, Nelson LM. Women with spontaneous 46,XX primary ovarian insufficiency (hypergonadotropic hypogonadism) have lower perceived social support than control women. Fertil Steril. 2009; 92(2): 688-93. doi: 10.1016/j.fertnstert.2008.07.1718. PMID: 18829005, PMCID: PMC2734403.

19) Dingley CE. Inner Strength as a Predictor of Quality Of Life and Self-Management in Women with Cancer. Ph.D. Thesis in Nursing. School of Nursing University of Colorado. 2008. Available from: http://search.proquest.com/docview/304338313.

20) Putnam KF. The relationship of inner strength and health promoting behaviors and their effect on quality of life in midlife women. Ph.D. Thesis in Nursing. School of Nursing. Virginia Commonwealth University. 2008.

21) Roux G, Dingley CE, Bush HA. Inner strength in women: Meta synthesis of qualitative findings in theory development. J Theory Constr Test. 2002; 6(1): 86-93. 
22) Rahnavard Z, Nodeh $\mathrm{ZH}$, Hatamipour K. Congestive heart failure: Predictors of health-related quality of life in Iranian women. Contemp Nurse. 2014; 47(1-2): 159-67. doi: 10.5172/conu.2014.47.1-2.159. PMID: 25267138 .

23) Mohammadi E, Khoshab H, Kazemnejad A. Activities of daily living for patients with chronic heart failure: a partnership care model evaluation. Appl Nurs Res. 2016; 30: 261-7. doi: 10.1016/j.apnr.2015.01.008. PMID: 27091288.

24) Mohammadi Zeidi I, Pakpour Hajiagha A, Mohammadi Zeidi B. Reliability and validity of Persian version of the health-promoting lifestyle profile. J Mazandaran Univ Med Sci. 2012; 21(1): 102-13.

25) Potter PA, Perry AG. Fundamental of Nursing. 7th edition Missouri; Mosby Elsevier. 2009.

26) Beal CC, Stuifbergen AK, Brown A. Predictors of a health promoting lifestyle in women with fibromyalgia syndrome. Psychol Health Med. 2009; 14(3): 343-53. doi: 10.1080/13548500902730093. PMID: 19444712.

27) Mirzaee A. Health Education and Health Promotion. Hatami H. (editor) Textbook of Public Health. 3rd edition. Volume 1. Tehran; Arjmand. 2009.

28) Khodadadi A, Sayadi A, Smaili H. Evolution of Knowledge of the principles of self-care in acute coronary syndrome patients admitted to Aliebn Abitaleb Rafsanjan university hospital during. Journal of Nursing, Midwifery and Paramedical of Rafsanjan. 2010; 5(1 and 2): 8-16.

29) Chun SY, Park H, Lee TH, Park EC. Do Long Term Cancer Survivors Have Better Health-Promoting Behavior than Non-Cancer Populations? Case-Control Study in Korea. Asian Pac J Cancer Prev. 2015; 16(4): 1415-20. PMID: 25743808. 